\title{
BMJ Open Protocol for assessing the determinants of preoperative test-ordering behaviour for low-risk surgical procedures using a theoretically driven, qualitative design
}

\author{
Andrea Pike (D) , ${ }^{1}$ Krista Mahoney, ${ }^{2}$ Andrea M Patey, ${ }^{3}$ Samantha Inwood (D) , \\ Sameh Mortazhejri, ${ }^{3}$ Rebecca Lawrence, ${ }^{1}$ Amanda Hall, ${ }^{1}$ De-implementing Wisely \\ Research Group
}

To cite: Pike A, Mahoney K, Patey AM, et al. Protocol for assessing the determinants of preoperative test-ordering behaviour for low-risk surgical procedures using a theoretically driven, qualitative design. BMJ Open 2020;10:e036511. doi:10.1136/ bmjopen-2019-036511

- Prepublication history and additional material for this paper are available online. To view these files, please visit the journal online (http://dx.doi. org/10.1136/bmjopen-2019036511).

Received 18 December 2019

Revised 31 March 2020 Accepted 03 April 2020

Check for updates

(C) Author(s) (or their employer(s)) 2020. Re-use permitted under CC BY-NC. No commercial re-use. See rights and permissions. Published by BMJ.

${ }^{1}$ Primary Healthcare Research Unit, Faculty of Medicine,

Memorial University of

Newfoundland, St. John's,

Newfoundland and Labrador, Canada

${ }^{2}$ Faculty of Medicine, Memorial University of Newfoundland,

St. John's, Newfoundland and Labrador, Canada

${ }^{3}$ Centre for Implementation Research, Ottawa Hospital Research Institute, Ottawa, Ontario, Canada

Correspondence to

Dr Amanda Hall;

amanda.hall@med.mun.ca

\section{ABSTRACT}

Introduction Current evidence suggests that preoperative tests such as chest $X$-rays, electrocardiograms and baseline laboratory studies may not be useful for healthy patients undergoing low-risk surgical procedures. Routine preoperative testing for healthy patients having low-risk surgery is not a scientifically sound practice. In this study, we will interview healthcare providers working at medical facilities where low-risk surgical procedures are carried out. This will allow us to gain insight into the determinants of preoperative testing behaviours for healthy patients undergoing low-risk surgeries and their barriers and enablers to guideline adherence.

Methods and analysis We will use semistructured interviews with anaesthesiologists, surgeons and preadmission clinic nurses to assess the determinants of preoperative testing behaviours. The interview guide was designed around the Theoretical Domains Framework (TDF), developed specifically to determine the barriers and enablers to implementing evidence-based guidelines. Interviews will be audio-recorded, transcribed verbatim and coded according to the TDF. Key themes will be generated for each of the identified domains.

Ethics and dissemination We have received ethics approval from the Health Research Ethics Board in Newfoundland and Labrador (HREB \#2018.190) for this study. The results of this work will be disseminated through a peer-reviewed publication, presentation at a healthcare forum and plain-language infographic summaries.

Additionally, deidentified data collected and analysed for this study will be available for review from the corresponding author on reasonable request.

\section{INTRODUCTION}

Preoperative testing (eg, chest X-rays, electrocardiograms (ECG), and baseline laboratory studies, such as bloodwork and urine analysis) is used to supplement the clinical history and physical examination findings of patients scheduled to receive anaesthesia. ${ }^{12}$ These tests are completed to provide additional information about high-risk patients (ie, those with known risk factors identified via their clinical history and physical examination) that will help
Strengths and limitations of this study

- This study is using the Theoretical Domains Framework synthesised from 33 behaviour change theories to guide the conduct and analysis of these interviews and ultimately form a theory-based intervention.

- The research team will use rigorous methods to collect and analyse the data.

- The team is using the Consolidated Criteria for Reporting Qualitative Research (COREQ) 32-item checklist to guide our methods and reporting.

- The findings of this study cannot be generalised or directly extrapolated to other settings.

anaesthesiologists prepare them for surgery and improve perioperative outcomes. ${ }^{23}$ In practice, however, preoperative testing has been implemented using a variety of strategies. Although there does not seem to be a common terminology, three different strategies are commonly referred to in the literature. The Agency for Healthcare Research and Quality refers to these strategies as:

1. Routine testing: tests are conducted on all patients undergoing given procedures, regardless of patient history.

2. Per protocol testing: tests are conducted on a predefined subset of patients undergoing a given procedure.

3. Ad hoc testing: preoperative testing is done at the discretion of the clinician doing a preoperative evaluation based on patients' clinical history and physical examination findings. ${ }^{4}$

Without supporting evidence, many hospitals have chosen to implement routine testing as a sort of 'fail-safe', seemingly under the assumption that more information (from more tests) will increase patient safety and decrease potential legal action resulting from adverse 
events. ${ }^{2}$ Several systematic reviews have been undertaken to search for evidence supporting this practice. Keay's 2012 Cochrane review ${ }^{5}$ found that routine preoperative tests (eg, ECG chest X-ray, bloodwork) for cataract surgery did not reduce the risk of intraoperative or postoperative adverse events when compared with selective or no testing. ${ }^{5}$ Similarly, another review, including three randomised controlled trials (two involving cataract surgeries and one including various types of ambulatory procedures) showed that routine preoperative testing did not affect clinical management or reduce morbidity or mortality compared with no testing, and importantly there was also no difference on the total perioperative complications or procedure cancellations between the two groups. ${ }^{46}$ For other types of low-risk surgeries, there are fewer studies on the beneficial or detrimental effects of routine testing or lack thereof making it difficult to make any conclusions about its utility in these contexts. ${ }^{47}$ However, it is important to remember that the scientific foundation for the implementation of routine preoperative testing is very weak. ${ }^{47}$ Many surgeons and anaesthesiologists also do not think that routine testing is useful. Instead, they report ordering preoperative tests because it is a part of the surgical protocol where they work, or because they think other members of the medical team require the tests to be completed or to avoid surgery delays or cancellations. ${ }^{89}$

Because of the lack of evidence supporting routine testing, clinical practice guidelines and Choosing Wisely recommendations advise against routine preoperative testing for healthy adult patients undergoing low-risk surgical procedures. ${ }^{10-14}$ Low-risk surgeries are those in which the combined surgical and patient characteristics predict a low risk (defined as $<1 \%$ in American guidelines) of a major adverse cardiac event such as death or myocardial infarction. ${ }^{15} 16$ In addition to the fact that it does not appear to be useful, routine testing is very costly. ${ }^{212}{ }^{17} \mathrm{In}$ Canada, preoperative testing is considered a major driver of the $30 \%$ of tests and treatments performed that are considered unnecessary. ${ }^{18}$ In the USA, an estimated $\$ 18$ billion is spent on preoperative testing every year. ${ }^{2}$ There is also reasonable speculation that benefits realised using routine testing do not outweigh the drawbacks which include extra testing, incidental findings that require additional costly investigations, surgical delays and patient stress $/$ harm. $^{2}$

However, despite recommendations to the contrary, routine preoperative testing for low-risk surgeries persists. Studies published in the last 5 years show that between the fiscal years 2011/2012 and 2012/2013, cardiac tests (ECG, chest X-ray, stress test or transthoracic echo) preceded $17.9 \%-35.5 \%$ of low-risk procedures across the Canadian provinces of Alberta, Saskatchewan and Ontario. ${ }^{11}$ Additionally, two studies in Ontario using provincial data from 2008 to 2013 showed that ECG and chest X-ray were conducted before $30.9 \%$ and $10.8 \%$ of procedures, respectively, and that preoperative bloodwork preceded $30.1 \%$ of low-risk procedures. ${ }^{19} 20$ Finally, a retrospective review of patients undergoing elective lumbar laminectomy between 2013 and 2017 by a single surgeon at Queen's University
Health Sciences Centre in Ontario found that $89.5 \%$ of patients underwent at least one preoperative blood test. ${ }^{21}$ Similar figures have been reported internationally. ${ }^{122-24}$ An assessment of data from Newfoundland's 10 surgical sites is underway and will indicate the scope of the problem in our local context.

\section{Improving the uptake of guidelines}

It is well accepted that simply disseminating guidelines is unlikely to change clinical practice behaviours. ${ }^{25}$ Increasingly, it is recognised that deimplementation might only be successful if we develop interventions that recognise and include provisions for the fact that any change in health professional behaviour must occur within complex (and often chaotic) healthcare environments. According to Grol and Wensings's model for behaviour change interventions, before we can change a behaviour, we need to understand why it is occurring. ${ }^{26} 27$ To do this, we must assess barriers and facilitators to performing the target behaviour and select behaviour change intervention strategies that will appropriately address those factors. Ideally, this process should be guided by a theoretical framework of established psychological theories of behaviour change. ${ }^{25-28}$

\section{Determinants of preoperative test-ordering behaviour}

Only one published study has previously investigated healthcare providers' barriers to deimplementing routine preoperative tests for healthy patients undergoing lowrisk surgery. Patey et al examined anaesthesiologists' and surgeons' perceptions about routine preoperative testing in low-risk patients in Ontario, Canada. ${ }^{8}$ The most common barriers they found were related to the lack of clarity regarding who was responsible for test ordering, perceived inability to cancel tests ordered by colleagues, and the fact that tests were being ordered and completed before the anaesthesiologists were able to examine the patient. ${ }^{8}$ Building on this work, we will investigate our local context by conducting a series of semistructured interviews with surgeons, anaesthesiologists, preoperative clinic nurses in NL. We will use the determinants identified through these interviews to inform the design of an intervention to optimise preoperative testing in NL.

\section{Objective}

The objective of this study is to identify barriers and facilitators to reducing unnecessary preoperative tests which will inform the development of an intervention to improve uptake of preoperative testing guidelines.

\section{METHODS}

\section{Patient and public involvement}

Patients were involved in developing the grant application supporting this work and will be involved in the interpretation and dissemination of the results.

\section{Design}

This is an exploratory, qualitative study describing healthcare provider experiences and practices related to preoperative evaluation. A framework analysis of the data will be used to clarify our understanding of healthcare providers' 
barriers and facilitators to deimplementing low-value preoperative testing.

\section{Participants}

Eligible participants are healthcare providers who are typically involved in ordering preoperative tests. In Newfoundland, this includes only surgeons, anaesthesiologists and preoperative clinic nurses. Thus, any surgeons, anaesthesiologists or preoperative clinic nurses practising in Newfoundland and Labrador (NL) who order preoperative tests for patients undergoing surgery are eligible to participate in this study. We recognise that different health providers may be responsible for preoperative test ordering in other jurisdictions which may limit the applicability of our results in those contexts. Purposive snowball sampling will be used to identify study participants. This sampling strategy is designed to encourage a wide variation of perspectives and ensure representation from across the four regional health authorities (RHAs) in Newfoundland \& Labrador, as well as a variety of surgical subspecialties. To do this, we will identify a key informant within the largest RHA, Eastern Health, to help provide an initial list of three potential participants as well as potential informants from the other three RHAs across the province. At the end of their interview, all participants will be asked to identify additional people who may be interested in participating in the study. To help ensure a diverse range of perspectives, we will also state that we are seeking participants who may have different views on this matter.

Based on Francis $e t a l$ s recommendations for sample size, we will conduct a minimum of 15 interviews, 5 from each profession. ${ }^{29}$ Once 15 interviews are completed, they will be analysed to determine if we have achieved for thematic saturation (the point at which no new information is offered) and geographic diversity (representation from each of the four provincial health authorities). If new themes arise in the last 3 interviews (across all groups) that have not been covered in the previous 12, or there is a lack of geographic representation, then we will conduct 3 additional interviews, repeating this iterative process either to saturation, or a maximum of 25 interviews. If themes are saturated at $\mathrm{n}=15$, then no further interviews will be sought. If during this process researchers find that there are stark differences between professions, then they will be analysed separately and thematic saturation will be assessed by profession, instead of as a whole.

\section{Data collection}

Following methods outlined in the TDF Guide and a barriers assessment with Ontario anaesthesiologists and surgeons, data will be collected using in-depth interviews. ${ }^{830}$ The relative lack of research in this area lends itself to the use of semistructured interviews which use a guided but still open approach that will allow participants to offer information beyond what is explicitly asked.

Potential participants (identified using our sampling strategy) will be emailed by a researcher ( $\mathrm{KM}$ or $\mathrm{AH})$ asking if they are interested in participating in a study investigating their views and test-ordering behaviours for healthy patients undergoing low-risk surgeries. Following the work of Patey $e t a l^{8}{ }^{8}$ we define healthy patients as those without additional medical conditions that could complicate anaesthesia management. If they are interested in volunteering, then they will be provided with additional information about the study and an interview time scheduled. Formal written consent is not required as consent is implied by the completion of the interview.

Interviews will be conducted by two healthcare researchers with experience in qualitative interviewing $(\mathrm{AP}, \mathrm{AH}$, both with graduate degrees in the health sciences) and may also include team members RL or KM to take field notes (all interviewers are women). Interviews will be conducted over the phone or in-person, whatever is private and most convenient for the participant. They are expected to take around 1 hour and participants will be provided a CAD $\$ 100$ gift card honorarium. All interviews will be audio-recorded and transcribed verbatim.

\section{Interview guide}

The interview guide (adapted from Patey et als study with surgeons and anaesthesiologists on preoperative testing in Ontario) was developed using the Theoretical Domains Framework (TDF). ${ }^{8}$ Please see the online supplementary appendix 1 for the complete interview guide. The TDF was originally developed by Michie et al to identify factors influencing health professional's implementation of evidencebased guidelines into practice. ${ }^{30-33}$ It was developed by synthesising over 128 key theoretical constructs from 36 behaviour change theories into overarching domains presented in a single framework. ${ }^{30}{ }^{33}$ Examples of domains include knowledge, skills, beliefs about capabilities and social influences. Each of the theoretical domains may be a determinant of the behaviour that requires change. Depending on the behaviour in question and contextual factors, some domains are likely to be more important than others. Once key domains are identified as barriers to changing a particular behaviour, strategies can be selected that best target those particular domains.

The TDF can be applied to identify which domains are key in changing a particular behaviour. For example, Atkins $e t$ al have produced a guide on how to apply the TDF to undertake the assessment of barriers and enablers to behaviour change. ${ }^{30}$ This is commonly achieved by using the TDF to develop qualitative data collection tools and methods of analysis. In the following paragraphs, we describe our process of applying the TDF to the development of our interview guide and data analysis.

The interview guide includes $1-4$ questions per domain (31 questions in total). It is intended to elicit participant beliefs about their behaviour through the lens of each domain. Prompts are provided in the interview guide to assist the interviewer in clarifying participants' responses if needed. The behaviour under investigation is ordering preoperative tests for healthy patients undergoing low-risk surgical procedures. Preoperative tests included in the interview guide were chest X-rays and ECGs. Low-risk surgeries include knee arthroscopy, laparoscopic cholecystectomy, 
cataract removal and similar types of surgeries. Healthy patients are defined in the guide as having no comorbidities or additional medical conditions (other than the surgical condition) that might complicate anaesthesia management and perioperative care. No changes were implemented after pilot testing with our key informant, a vascular surgeon. Interview participants were instructed to base their answers on low-risk surgeries for healthy patients.

\section{Data analysis}

To begin, coders will read and reread the transcripts to become familiar with the data; they will begin coding after we have completed and transcribed approximately six interviews. We will analyse the data using a three-step process: (1) domain coding, (2) generating themes and (3) identifying key domains.

\section{Step 1: domain coding}

Two coders (SI, SM) have been trained to code the transcripts using the TDF coding scheme for this study. Under the guidance of an expert in TDF interviewing and coding (AMP), they practised coding transcripts from a previous, similar study. From this work, they created a codebook (using examples from their practice transcripts) that will serve as a guideline and reference for the coders to ensure accuracy and consistency when coding begins.

The codebook contains (1) the coding strategy and (2) a table of coded text. The coding strategy provides the coders an explicit method for deciding whether to code a section of the text, how much of the text to code, how to decide what domain to code it under, and how to handle disagreements. The table of the coded text includes the domains, their definitions and subconstructs, examples of codes for each domain, as well as the rationale for why each text was coded in that domain. Examples that clearly exemplify a domain or that elicited confusion or discussion during practice coding were selected. After they being coding, the coders will update the codebook as appropriate, including adding new examples of coded text. Please see the online supplementary appendix 2 for the codebook we developed during the training exercise.

SI and SM will code all transcripts in NVivo V.12 using the codebook described earlier. They will code one pilot interview simultaneously to consensus, with access to an expert coder (AMP) as needed. Using the second pilot interview, the researchers will code independently and calculate the Fleiss kappa ( $\kappa)$ for all domains to assess how reliably they coded the same statement to the same domain, thereby validating the coding strategy outlined in the codebook. Any domains with $\kappa<0.8$ will be coded to consensus. If the researchers are confident in their strategy at this point, then they will begin coding independently, reviewing their kappas every three interviews to ensure they are remaining consistent. Again, any domains with $\kappa<0.8$ will be reviewed and coded to consensus.

\section{Step 2: generating themes}

After the data (quotes from interviews) are coded into TDF domains, we will use a process of open coding to generate subthemes which will then be reviewed to create broader, overarching themes. First one coder will review all passages coded into each domain, generating a sentence for each passage that reflects the key message related to the coded domain. This will result in a set of subthemes that represent an important belief about a barrier or enabler to the target behaviour (eg, lack of knowledge about guidelines, worry about missing underlying problem). ${ }^{84}$ These subthemes will be refined as we move through the analytic process for all of our data to reflect common subthemes across all participants' responses. In some cases, some subthemes will likely will be pulled directly from particularly expressive quotes/passages.

Next, we will examine the subthemes at each domain to identify broader themes or patterns in the data. For example, subthemes of all the negative consequences described by participants (eg, worry about missing underlying problems, patient upset) will be grouped into a broader theme called negative consequences. At the end of this exercise, we will have a list of broad themes that reflect the overall barriers and enablers to following evidence-based guidelines for preoperative testing and subthemes that provide more specific details about these barriers and facilitators. All themes and subthemes with supporting quotes will be reviewed by the second coder and a health psychologist. During this process, we will consider whether the themes and subthemes are supported by data (ie, do they make sense) and if there is remaining overlap among the themes.

\section{Step 3: identifying key domains}

Key domains will be identified through consensus discussion between the two researchers responsible for coding (SM, SI), confirmed by a health psychologist (AMP), and subsequently reviewed with the larger research team. Key domains refer to those domains which provide sufficient evidence to target in an intervention for behaviour change. ${ }^{35}$ Briefly, three factors will be considered to identify key domains: (1) reported strength of opinion that the beliefs influenced the behaviour, (2) presence of conflicting beliefs and (3) frequency of the beliefs across interviews. ${ }^{30}$ All of these factors will be considered concurrently to establish domain importance. At this stage, we will also ensure that the themes identified reflect a common view across multiple participants in the context of the entire dataset.

\section{ETHICS AND DISSEMINATION}

Ethics approval for this study was granted by the Health Research Ethics Board in Newfoundland and Labrador (HREB \#2018.190). Study results will be disseminated through a peer-reviewed manuscript publication, presentation at a healthcare forum, and plain language infographic summaries suitable for all audiences. Additionally, deidentified data collected and analysed for this study will be available for review from the corresponding author on reasonable request.

Acknowledgements The authors formally acknowledge the contributions of the De-implementing Wisely Research Group to the conceptualisation and design of this work. 
Collaborators De-implementing Wisely Research Group:Jeremy Grimshaw; Wendy Levinson; Kyle Kirkham; Shawn Dowling; Kelly Mrklas; Justin Presseau; Sacha Bhatia; Todd Sikorski; Patrick Parfrey; Yamile Jasaui.

Contributors $\mathrm{AH}, \mathrm{AMP}, \mathrm{KM}$ and the remaining members of the De-implementing Wisely Research Group: conceptualised and designed this barriers assessment. AP, KM, SI, AH: drafted the protocol. AH, AMP, KM, RL: developed and reviewed the question guide. AMP, SM, AH, SI, RL: developed the codebook. AP, AMP, AH, KM, SI: provided feedback on both content and clarity. All authors reviewed the manuscript and provided feedback and provided their approval to publish the manuscript.

Funding This work was supported by the Canadian Institute of Health Research, grant number 398527.

\section{Competing interests None declared.}

Patient and public involvement Patients and/or the public were involved in the design, or conduct, or reporting, or dissemination plans of this research. Refer to the Methods section for further details.

\section{Patient consent for publication Not required}

Provenance and peer review Not commissioned; externally peer reviewed.

Open access This is an open access article distributed in accordance with the Creative Commons Attribution Non Commercial (CC BY-NC 4.0) license, which permits others to distribute, remix, adapt, build upon this work non-commercially, and license their derivative works on different terms, provided the original work is properly cited, appropriate credit is given, any changes made indicated, and the use is non-commercial. See: http://creativecommons.org/licenses/by-nc/4.0/.

\section{ORCID iDs}

Andrea Pike http://orcid.org/0000-0003-4020-2291

Samantha Inwood http://orcid.org/0000-0001-8900-9017

\section{REFERENCES}

1 Merchant R, Chartrand D, Dain S, et al. Guidelines to the practice of anesthesia - revised edition 2016. Can J Anesth/J Can Anesth 2016;63:86-112.

2 Kumar A, Srivastava U. Role of routine laboratory investigations in preoperative evaluation. J Anaesthesiol Clin Pharmacol 2011:27:174-9.

3 Valchanov KP, Steel A. Preoperative investigation of the surgical patient. Surgery 2008;26:363-8.

4 Balk EM, Earley A, Hadar N, et al. Benefits and Harms of Routine Preoperative Testing: Comparative Effectiveness. Rockville, MD: Agency for Healthcare Research and Quality, 2014. www. effectivehealthcare.ahrq.gov/reports/final.cfm

5 Keay L, Lindsley K, Tielsch J, et al. Routine preoperative medical testing for cataract surgery. Cochrane Database Syst Rev 2009;2.

6 Johansson T, Fritsch G, Flamm M, et al. Effectiveness of non-cardiac preoperative testing in non-cardiac elective surgery: a systematic review. Br J Anaesth 2013;110:926-39.

7 Czoski-Murray C, Jones ML, McCabe C, et al. Routine pre-operative testing regression analysis report. In: What Is the Value of Routinely Testing Full Blood Count, Electrolytes and Urea, and Pulmonary Function Tests Before Elective Surgery in Patients with No Apparent Clinical Indication and in Subgroups of Patients with Common Comorbidities: A Systematic Review of the Clinical and CostEffective Literature. NIHR Journals Library 2012.

8 Patey AM, Islam R, Francis JJ, et al. Anesthesiologists' and surgeons' perceptions about routine pre-operative testing in low-risk patients: application of the theoretical domains framework (TDF) to identify factors that influence physicians' decisions to order preoperative tests. Implement Sci 2012;7:1748-5908-7-52.

9 Bass EB, Steinberg EP, Luthra R, et al. Do ophthalmologists, anesthesiologists, and internists agree about preoperative testing in healthy patients undergoing cataract surgery? Arch Ophthalmol 1995:113:1248-56.

10 Benarroch-Gampel J, Sheffield KM, Duncan CB, et al. Preoperative laboratory testing in patients undergoing elective, low-risk ambulatory surgery. Ann Surg 2012;256:518-28.

11 Bouck Z, Pendrith C, Chen X-K, et al. Measuring the frequency and variation of unnecessary care across Canada. BMC Health Serv Res 2019;19:446
12 Colla $\mathrm{CH}$, Morden NE, Sequist TD, et al. Choosing wisely: prevalence and correlates of low-value health care services in the United States. $J$ Gen Intern Med 2015;30:221-8.

13 Coffman J, Tran T, Quast T, et al. Cost conscious care: preoperative evaluation by a cardiologist prior to low-risk procedures. BMJ Open Qual 2019;8:e000481.

14 van Klei WA, Bryson GL, Yang $\mathrm{H}$, et al. The value of routine preoperative electrocardiography in predicting myocardial infarction after noncardiac surgery. Ann Surg 2007;246:165-70.

15 Duceppe E, Parlow J, MacDonald P, et al. Canadian cardiovascular Society guidelines on perioperative cardiac risk assessment and management for patients who undergo noncardiac surgery. Can $J$ Cardiol 2017;33:17-32.

16 Fleisher LA, Fleischmann KE, Auerbach AD, et al. 2014 ACC/ AHA guideline on perioperative cardiovascular evaluation and management of patients undergoing noncardiac surgery: a report of the American College of Cardiology/American heart association Task force on practice guidelines. J Am Coll Cardiol 2014;64:e77-137.

17 Chen CL, Clay TH, McLeod S, et al. A revised estimate of costs associated with routine preoperative testing in Medicare cataract patients with a Procedure-Specific indicator. JAMA Ophthalmol 2018;136:231-8

18 Levinson W, O'Toole D. Unnecessary care in Canada, 2017. Available: http://www.deslibris.ca/ID/10090639 [Accessed 12 Dec 2019].

19 Kirkham KR, Wijeysundera DN, Pendrith C, et al. Preoperative laboratory investigations: rates and variability prior to low-risk surgical procedures. Anesthesiology 2016;124:804-14.

20 Kirkham KR, Wijeysundera DN, Pendrith C, et al. Preoperative testing before low-risk surgical procedures. CMAJ 2015;187:E349-58.

21 Tohidi M, Lung T, Yen D. Routine perioperative practices and postoperative outcomes for elective lumbar laminectomies. J Spine Surg 2018;4:588-93.

22 Onuoha OC, Hatch MB, Miano TA, et al. The incidence of unindicated preoperative testing in a tertiary academic ambulatory center: a retrospective cohort study. Perioper Med 2015;4.

23 Sui W, Theofanides MC, Matulay JT, et al. Utilization of preoperative laboratory testing for low-risk, ambulatory urologic procedures. Urology 2016;94:77-84.

24 Yonekura $\mathrm{H}$, Seto K, Ide K, et al. Preoperative blood tests conducted before low-risk surgery in Japan: a retrospective observational study using a nationwide insurance claims database. Anesth Analg 2018;126:1633-40.

25 Grimshaw JM, Thomas RE, MacLennan G, et al. Effectiveness and efficiency of guideline dissemination and implementation strategies. Health Technol Assess 2004;8:1-72.

26 Grol R, Wensing M. Effective implementation: a model. Improv Patient Care Implement Change Clin Pract 2005;41.

27 Grol R, Grimshaw J. From best evidence to best practice: effective implementation of change in patients' care. Lancet 2003;362:1225-30

28 Improved clinical effectiveness through behavioural Research Group (iceberg). Designing theoretically-informed implementation interventions. Implement Sci 2006;1.

29 Francis JJ, Johnston M, Robertson C, et al. What is an adequate sample size? Operationalising data saturation for theory-based interview studies. Psychol Health 2010;25:1229-45.

30 Atkins L, Francis J, Islam R, et al. A guide to using the theoretical domains framework of behaviour change to investigate implementation problems. Implement Sci 2017;12:77.

31 Cane J, O'Connor D, Michie S. Validation of the theoretical domains framework for use in behaviour change and implementation research. Implement Sci 2012;7:37.

32 Michie S, Johnston M, Abraham C, et al. Making psychological theory useful for implementing evidence based practice: a consensus approach. Qual Saf Health Care 2005;14:26-33.

33 Michie S, Johnston M, Francis J, et al. From theory to intervention: mapping theoretically derived behavioural determinants to behaviour change techniques. Appl Psychol 2008:57:660-80.

34 Francis JJ, Stockton C, Eccles MP, et al. Evidence-Based selection of theories for designing behaviour change interventions: using methods based on theoretical construct domains to understand clinicians' blood transfusion behaviour. Br J Health Psychol 2009;14:625-46.

35 Patey AM, Curran JA, Sprague AE, et al. Intermittent auscultation versus continuous fetal monitoring: exploring factors that influence birthing unit nurses' fetal surveillance practice using theoretical domains framework. BMC Pregnancy Childbirth 2017;17:320. 